\title{
Effect of GH replacement therapy in two male siblings with combined $X$-linked hypophosphatemia and partial GH deficiency
}

\author{
Snjezana M Schütt, Marius Schumacher, Paul M Holterhus, Stefanie Felgenhauer and Olaf Hiort \\ Department of Pediatrics, University of Lübeck, Ratzeburger Allee 160, 23538 Lübeck, Germany \\ (Correspondence should be addressed to O Hiort; Email: hiort@paedia.ukl.mu-luebeck.de)
}

\begin{abstract}
Objective: X-linked hypophosphatemia (XLH) is characterized by low serum phosphorus, relative 1,25dihydroxyvitamin $\mathrm{D}_{3}$ deficiency and rickets. It is caused by mutations in the phosphate-regulating gene with homologies to endopeptidases on the X chromosome (PHEX). The conventional treatment of XLH includes the administration of phosphate and calcitriol; however, treated patients usually present with a short stature. Therefore, additional coexistent defects, such as GH deficiency, are under debate. Patients and methods: Two male siblings presented with a disproportionate growth failure and rickets. Investigation of calcium and phosphate metabolism, molecular genetic analysis of the PHEX gene and GH function tests were initiated.

Results: Both patients showed typical clinical and biochemical signs of XLH. Molecular genetic analysis revealed a 747 CGA (Arg)-TGA (End) mutation in exon 22 of the PHEX gene, confirming XLH. Since treatment with phosphate and calcitriol alone failed to improve growth in both patients, the GH axis was examined and a partial GH deficiency was diagnosed in both cases. Almost 3 years of additional therapy with recombinant human $\mathrm{GH}$ (rhGH) led to a significant improvement of height standard deviation scores (HtSDS).

Conclusions: Poor growth in XLH may, in at least some patients, be aggravated by GH deficiency. Hence, GH deficiency should be considered in extremely poorly growing patients with XLH, because these patients are likely to benefit from rhGH therapy.
\end{abstract}

European Journal of Endocrinology 149 317-321

\section{Introduction}

$\mathrm{X}$-linked hypophosphatemia (XLH) is the most common form of hereditary phosphopenic rickets (1). The disorder is attributed to renal phosphate wasting and is characterized by low serum phosphorus, normal parathyroid hormone levels and inappropriately normal 1,25-dihydroxyvitamin $\mathrm{D}_{3} \quad\left(1,25-(\mathrm{OH})_{2} \mathrm{D}_{3}\right)$ concentrations (2). The phenotype of affected patients is variable and usually includes rachitic and osteomalacic bone disease, poor dental development and growth retardation (2). Conventional treatment of XLH is based on the combined administration of 1,25$(\mathrm{OH})_{2} \mathrm{D}_{3}$ (calcitriol) and oral phosphate salts $(3,4)$. This therapy is known to improve bone mineralization and rickets, particularly if started as soon as possible after birth (5). However, linear growth of treated patients often remains poor $(6,7)$. Therefore, additional defects, such as growth hormone (GH) deficiency, have been discussed (8). We present two male siblings with molecular genetic proven XLH and partial $\mathrm{GH}$ deficiency, who showed improved linear growth under a recombinant human $\mathrm{GH}(\mathrm{rhGH})$ replacement therapy.

\section{Case reports}

\section{Clinical and laboratory data at admission}

Two male siblings presented to our hospital at the age of 28 (patient A) and 14 (patient B) months, because of a blunted growth, bowing of the long bones and a waddling gait. In addition, both patients suffered from recurrent dental abscesses. The family history was positive as the mother suffered from rickets and disproportionate growth failure with a final height of $150 \mathrm{~cm}$. Clinical examination of the two boys revealed negative height S.D. scores (HtSDS) and an elevated upper segment/lower segment (US/LS) ratio (Table 1). Both patients showed radiological signs of rickets with metaphyseal widening, frying and cupping of the ulna, distal femur and bow legs. Laboratory investigations were performed as described (9) and revealed 
Table 1 Clinical findings and laboratory investigation of calcium and bone metabolism in patients $A$ and $B$ at admission (without therapy), after conventional therapy alone (1,25-dihydroxyvitamin $D_{3}(V i t D)+$ phosphate $\left.(P)\right)$ and after 3 years of combined conventional and rhGH replacement therapy (VitD $+\mathrm{P}+\mathrm{rhGH})$.

\begin{tabular}{|c|c|c|c|c|}
\hline & Patient & Without therapy & VitD+P & VitD+P+rhGH \\
\hline \multirow[t]{2}{*}{ Chronological age (years) } & A & 2.4 & 5.6 & 7.7 \\
\hline & B & 1.2 & 4.8 & 6.0 \\
\hline \multirow[t]{2}{*}{ Bone age (years) } & A & 2.0 & 3.3 & 5.9 \\
\hline & B & 1.0 & 3.0 & 4.6 \\
\hline \multirow[t]{2}{*}{ HtSDS } & A & -3.8 & -4.8 & -3.3 \\
\hline & B & -1.9 & -3.0 & -1.5 \\
\hline \multirow[t]{2}{*}{ US/LS ratio } & A & 1.52 & 1.30 & 1.03 \\
\hline & B & 1.31 & 1.27 & 1.23 \\
\hline \multirow[t]{2}{*}{ IGF-I (17-248 $\mu \mathrm{g} / \mathrm{I})$} & A & n.d. & 90 & 387 \\
\hline & B & n.d. & 90 & 349 \\
\hline \multirow[t]{2}{*}{ Calcium (8.4-10.6 mg/dl) } & A & 9.8 & 10 & 9.4 \\
\hline & B & 9.2 & 9.8 & 9.2 \\
\hline \multirow[t]{2}{*}{ Phosphate $(4-6 \mathrm{mg} / \mathrm{dl})$} & A & 2.4 & 3.8 & 3.9 \\
\hline & B & 3.0 & 3.5 & 4.1 \\
\hline \multirow[t]{2}{*}{ iPTH (15-55 pg/ml) } & A & 33 & 15 & 14 \\
\hline & B & 44 & 14 & 15 \\
\hline \multirow[t]{2}{*}{ AP $(200-600 \mathrm{U} / \mathrm{l})$} & A & 683 & 426 & 462 \\
\hline & B & 668 & 404 & 408 \\
\hline \multirow[t]{2}{*}{ TRP (85-98\%) } & A & 80.5 & 60.3 & 65.7 \\
\hline & B & 63.8 & 51.6 & 70.0 \\
\hline \multirow[t]{2}{*}{ TmP/GFR (4-8 mg/dl) } & A & 2.0 & 2.3 & 2.6 \\
\hline & B & 1.9 & 1.8 & 2.9 \\
\hline \multirow[t]{2}{*}{$\mathrm{U}-\mathrm{OHP} / \mathrm{Cr}(82-218 \mu \mathrm{g} / \mathrm{mg})$} & A & 233 & 118 & $94(60-160)^{\star}$ \\
\hline & B & $333(99-335)^{*}$ & 210 & 98 \\
\hline \multirow[t]{2}{*}{$\mathrm{U}-\mathrm{Ca} / \mathrm{Cr}(\mu \mathrm{g} / \mathrm{mg})$} & A & $68(11-188)^{\star}$ & $187(20-400)^{*}$ & $117(5-174)^{*}$ \\
\hline & B & $39(11-188)^{*}$ & $202(20-500)^{\star}$ & $92(10-300)^{\star}$ \\
\hline
\end{tabular}

$\mathrm{U}-\mathrm{OHP} / \mathrm{Cr}$, urinary hydroxyproline in relation to creatinine; $\mathrm{U}-\mathrm{Ca} / \mathrm{Cr}$, urinary calcium in relation to creatinine.

*The (age-dependent) normal range values are indicated in parentheses. n.d., not determined.

a normal serum calcium, a low serum phosphorus, a slightly elevated alkaline phosphatase (AP) and a normal intact parathyroid hormone (iPTH) concentration (Table 1). Tubular re-absorption of phosphate (TRP) and the tubular maximum rate of phosphate re-absorption in relation to the glomerular filtration rate $(\mathrm{TmP} / \mathrm{GFR})$ were decreased in both cases (Table 1). The urinary calcium/creatinine ratio was in the normal range, whereas the urinary total hydroxyproline/creatinine ratio was increased (Table 1).

\section{Molecular analysis of the PHEX gene}

In order to confirm the diagnosis of XLH, a molecular genetic analysis of the PHEX gene was performed. Genomic DNA was isolated from blood leucocytes by standard procedures. Exons 1-22 of the PHEX gene were individually amplified by polymerase chain reaction with specific primers. Subsequently, amplified products were analyzed by non-isotopic single strand conformation analysis (SSCA) (10). The exon displaying an aberrant electrophoretic pattern on SSCA was directly sequenced employing CY5-labeled primers on an automated sequencer (ALF Express, Pharmacia, Freiburg, Germany) according to the specifications of the manufacturer (11). With this approach, a cytosine to thymine substitution in exon 22 was detected in both patients, replacing arginine (CGA) in codon position 747 by a premature termination codon (TGA).

\section{Clinical and laboratory data after conventional therapy}

After conventional treatment for 4 and 3 years respectively, both patients had improved clinical and laboratory findings with normocalcemia, normal iPTH levels and normal AP (Table 1). The serum phosphate was slightly increased and the urinary hydroxyproline normalized, whereas TRP and TmP/GFR remained unchanged in both cases (Table 1). The radiological signs of rickets also improved; however, linear growth remained poor in both patients $(-4.8 \mathrm{HtSDS}$ in patient A and -3.0 HtSDS in patient B, Table 1).

\section{Evaluation of GH secretion}

Serum levels of insulin-like growth factor-I (IGF-I) and IGF-binding protein-3 (IGFBP-3) were assayed in duplicate in blood serum using a radioimmunoassay (Nichols Institute Diagnostics, San Juan Capistrano, CA, USA). Both IGF-I and IGFBP-3 were in the lower normal range in both patients (Table 2). Therefore, spontaneous $\mathrm{GH}$ secretion and $\mathrm{GH}$ stimulation tests were performed as described (12). Serum GH levels were measured in duplicate by an IRMA, using a commercially available 
Table 2 Evaluation of GH secretion.

\begin{tabular}{lccc}
\hline & Patient A $(\mu \mathrm{g} / \mathrm{l})$ & Patient B $(\mu \mathrm{g} / \mathrm{l})$ & Normal range $(\mu \mathrm{g} / \mathrm{l})$ \\
\hline IGF-I & 90 & 90 & $12-248$ \\
IGFBP-3 & 1.5 & 1.3 & $1.2-3.1$ \\
GH peaks at night & $5.3 / 6.9 / 4.2$ & $7.2 / 6.2 / 6.7$ & Three GH peaks $>10$ \\
GH peak after ITT & 7.1 & 6.0 & $\mathrm{GH}$ peak $>10$ \\
GH peak after AST & 7.2 & 7.1 & $\mathrm{GH}$ peak $>10$ \\
GH peak after GHRH test & 33 & 34 & $\mathrm{GH}$ peak $>10$ \\
\hline
\end{tabular}

ITT, insulin tolerance test; AST, arginine stimulation test.

kit (Nichols Institute Diagnostics). The spontaneous nightly $\mathrm{GH}$ secretion and the response of $\mathrm{GH}$ to at least two provocative pharmacological stimuli were inadequate in both patients (Table 2). The GH-releasing hormone $(\mathrm{GHRH})$ test revealed a normal $\mathrm{GH}$ response to GHRH (Table 2), indicating a hypothalamic defect. Thus, both siblings were diagnosed as having partial $\mathrm{GH}$ deficiency (12) and a rhGH replacement therapy was initiated.

\section{Clinical and laboratory data after 3 years of conventional treatment and additional rhGH replacement therapy}

A total of $0.03 \mathrm{mg} / \mathrm{kg}$ per day rhGH (Humatrope, Lilly, Bad Homburg, Germany) was administered by daily subcutaneous injections in the evening for 3 years. Both patients continued to receive conventional therapy with phosphate and calcitriol. Under this therapy an improvement in HtSDS $(-3.3$ in patient $A$ and -1.5 in patient $B$, Table 1 ) and linear growth (Fig. 1) was observed in both patients. In addition, both patients showed an increased annual growth velocity (from below the third percentile to the 75-90th percentile in patient $A$ and from the third percentile to the 75 th percentile in patient B, Table 3), which was most pronounced during the first treatment year and slowed down over the next 2 years (Table 3). The US/LS ratio decreased slightly in both patients, which was consistent with an improved clinical bowing (Table 1). Bone age remained retarded in both patients (Table 1). Improvement in height was accompanied by an increase in serum IGF-I levels, whereas only small changes of metabolic markers were observed and serum calcium, AP and iPTH levels remained within the normal range. After 3 years of rhGH therapy the serum phosphate remained unchanged in patient $A$ and slightly increased in patient B (Table 3). During the first year of rhGH therapy TRP increased significantly in patient $A$ and decreased in patient B (Table 3 ). Over the period of 3 years this parameter increased in both patients, but remained below the normal range (Table 3). The urinary calcium/creatinine ratio decreased in both patients and was within the normal range after 3 years of additional rhGH therapy (Table 3 ).
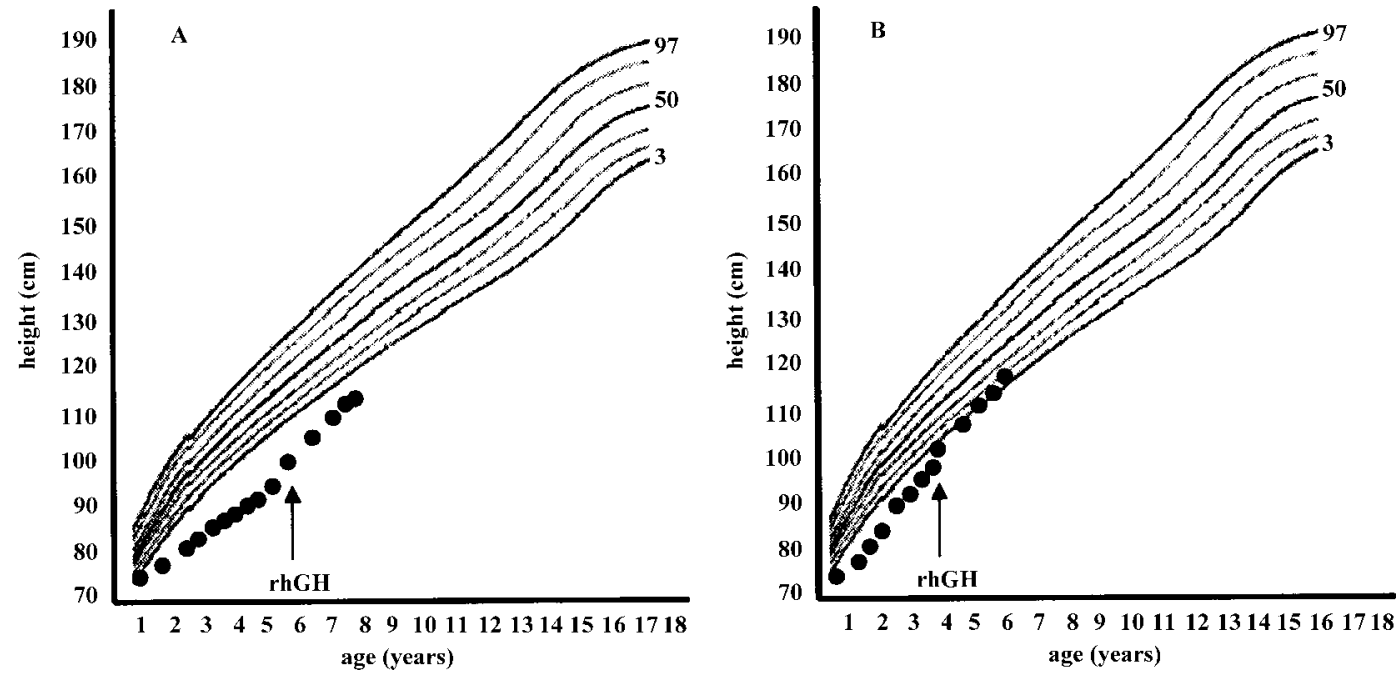

Figure 1 Growth charts of patients A and B before and under additional therapy with rhGH. The onset of the additional rhGH therapy is indicated by an arrow. 
Table 3 Kinetic response of laboratory parameters to $\mathrm{GH}$ therapy in comparison to annual growth velocity in patients $\mathrm{A}$ and $\mathrm{B}$ at the beginning of combined conventional and rhGH replacement therapy (start 1,25-dihydroxyvitamin $D_{3}(V i t D)+$ phosphate $(P)+$ rhGH), after 1 year (1 year VitD+ $\mathrm{P}+$ rhGH), 2 years (2 years VitD+ $\mathrm{P}+\mathrm{rhGH}$ ) and 3 years (3 years VitD+ $\mathrm{P}+\mathrm{rhGH})$ of combined conventional and $\mathrm{rhGH}$ replacement therapy.

\begin{tabular}{|c|c|c|c|c|c|}
\hline & Patient & Start VitD+P+rhGH & 1 year VitD + P+ rhGH & 2 years VitD+ $P+r h G H$ & 3 years VitD $+P+$ rhGH \\
\hline $\begin{array}{l}\text { Growth velocity } \\
\text { (cm/year) }\end{array}$ & $\begin{array}{l}\mathrm{A} \\
\mathrm{B}\end{array}$ & $\begin{array}{l}6.6(25 \text { th } P)^{*} \\
7.3(25 \text { th } P)\end{array}$ & $\begin{array}{c}8.8(97 \text { th } P)^{*} \\
9.9(>97 \text { th } P)^{*}\end{array}$ & $\begin{array}{c}6.1(50 \text { th } P)^{*} \\
6.2(25 \text { th }-50 \text { th } P)^{*}\end{array}$ & $\begin{array}{c}6.5(75 \text { th }-90 \text { th } P)^{*} \\
7.0(75 \text { th } P)^{\star}\end{array}$ \\
\hline Phosphate & $A$ & 3.8 & 3.7 & 3.8 & 3.9 \\
\hline$(4-6 \mathrm{mg} / \mathrm{dl})$ & $\mathrm{B}$ & 3.5 & 3.7 & 3.9 & 4.1 \\
\hline TRP & A & 60.3 & 68.3 & 62.0 & 65.7 \\
\hline$(85-98 \%)$ & $\mathrm{B}$ & 51.6 & 44.4 & 65.0 & 70.0 \\
\hline $\mathrm{U}-\mathrm{Ca} / \mathrm{Cr}$ & $A$ & $187(20-400)^{*}$ & $86.5(5-174)^{\star}$ & $127(5-174)^{\star}$ & $117(5-174)^{*}$ \\
\hline$(\mu \mathrm{g} / \mathrm{mg})$ & $\mathrm{B}$ & $202(20-500)^{\star}$ & $84.4(40-410)^{*}$ & $119(10-300)^{*}$ & $92(10-300)^{*}$ \\
\hline
\end{tabular}

$\mathrm{U}-\mathrm{Ca} / \mathrm{Cr}$, urinary calcium in relation to creatinine.

* The (age-dependent) normal range values and percentiles $(P)$ are indicated in parentheses.

\section{Discussion}

In both patients reported here with XLH and partial GH deficiency, linear growth remained poor under conventional treatment with phosphate and calcitriol alone, but clearly improved after initiation of a $\mathrm{GH}$ replacement therapy.

Due to the severe growth failure in both patients, evaluation of $\mathrm{GH}$ secretion was initiated and subsequently revealed the diagnosis of a partial $\mathrm{GH}$ deficiency. So far, defects in GH secretion have been limited to single case reports in $\mathrm{XLH}(5,7,13)$, and the majority of poorly growing patients with XLH seem to have normal parameters for $\mathrm{GH}$ secretion $(5,7,13-$ 16). However, our findings suggest that GH deficiency should be considered in at least some patients with XLH. The mechanism of GH deficiency in our patients remains unclear. In concordance with the report of Burgueno et al. (17), we found a normal GH response to GHRH in the GHRH test while the $\mathrm{GH}$ response to provocative stimuli was inadequate, suggesting a hypothalamic defect in both patients. Since a growth-controlling gene has been mapped to the same region as the PHEX gene (18), one may also hypothesize that at least some patients with XLH and severe growth failure may suffer from a concomitant genetic alteration of the growth-controlling gene. However, further studies are needed to investigate the mechanism of $\mathrm{GH}$ deficiency in such patients.

Recent studies suggest that GH could further improve calcium phosphate metabolism $(16,19,20)$ and linear growth in XLH $(14,19)$. Therefore, GH therapy in XLH has been investigated in a few small cohorts regardless of a $\mathrm{GH}$ secretion defect. At least in some patients, improvement of metabolic markers of calcium phosphate metabolism, growth rate and bone mineral density have been reported $(5,6,15,16,21,22,23)$. However, the effects of an adjunctive GH therapy on linear growth are difficult to evaluate because of the small sample sizes and the variability of diagnostic steps and treatment regimens. In our patients, rhGH was administered at a replacement dosage. Under this therapy, both the linear growth and the annual growth velocity improved significantly in both patients without advancement of bone age. In contrast to the study of Haffner et al. (7), the additional rhGH treatment did not aggravate the pre-existing US/LS disproportion in our patients. In agreement with the study of Baroncelli et al. (23), we observed an increase of phosphate levels in patient $B$ and an increase of TRP in both patients after 3 years of additional rhGH therapy. The metabolic markers of calcium phosphate metabolism remained largely unchanged. Concern has been raised that rhGH may induce hypercalciuria during calcitriol treatment (24). However, in our study rhGH did not increase urinary calcium excretion, which is consistent with the findings of Haffner et al. (7). We assume that impaired $\mathrm{GH}$ secretion may contribute to growth failure in at least some XLH patients and that these patients are likely to benefit from rhGH substitution. However, whether GH will generally have a positive effect in patients with XLH cannot be concluded from our data.

In conclusion, patients with XLH and severe growth failure should be tested for functional defects in $\mathrm{GH}$ secretion. For a comprehensive evaluation of the benefit of rhGH therapy in XLH individuals, prospective investigations of larger cohorts are necessary. In addition to the clinical parameters of growth, these studies should also include proper and regular analysis of calcium, phosphate and bone metabolism, determination of $\mathrm{GH}$ secretion as well as an analysis of the underlying molecular genetic alteration.

\section{Acknowledgements}

This work is dedicated to the late Professor Klaus Kruse for his detailed advice in the diagnosis and therapy of the patients. Moreover, we thank Gudrun Pauch and Mechthild Schumann for performing the biochemical measurements, and Andrea Lehners and Dagmar Struve for performing the molecular genetic analysis. 


\section{References}

1 Saggese G, Baroncelli GI, Bertelloni S \& Perri G. Growth hormone secretion in poorly growing children with renal hypophosphataemic rickets. European Journal of Pediatrics 1994153 548-555.

2 Saggese G, Baroncelli GI, Bertelloni S \& Perri G. Long-term growth hormone treatment in children with renal hypophosphatemic rickets: effects on growth, mineral metabolism, and bone density. Journal of Pediatrics 1995127 395-402.

3 Wilson DM, Lee PD, Morris AH, Reiter EO, Gertner JM, Marcus R et al. Growth hormone therapy in hypophosphatemic rickets. American Journal of Diseases of Children 1991145 1165-1170.

4 Seikaly MG, Brown R \& Baum M. The effect of recombinant human growth hormone in children with X-linked hypophosphatemia. Pediatrics $1997100879-884$.

5 Kruse K. Hinkel GK \& Griefahn B. Calcium metabolism and growth during early treatment of children with X-linked hypophosphataemic rickets. European Journal of Pediatrics 1998157 894-900.

6 Patel L, Clayton PE, Brain C, Pelekouda E, Addison GM, Price DA et al. Acute biochemical effects of growth hormone treatment compared with conventional treatment in familial hypophosphataemic rickets. Clinical Endocrinology $1996 \mathbf{4 4} 687-696$.

7 Haffner D, Wuhl E, Blum WF, Schaefer F \& Mehls O. Dysproportionate growth following long-term growth hormone treatment in short children with X-linked hypophosphaetmia. European Journal of Pediatrics $1995 \mathbf{1 5 4}$ 610-613.

8 Henneman PH, Forbes AP, Moldawer M, Dempsey EF \& Carrol EL. Effects of human growth hormone in man. Journal of Clinical Investigation 196039 1223-1238.

9 Wilson DM. Growth hormone and hypophosphatemic rickets. Journal of Pediatric Endocrinology and Metabolism 200013 993-998.

10 Hiort O, Sinnecker GHG, Willenbring H, Lehners A, Zöllner A \& Struve D. Nonisotopic single strand conformation analysis of the 5 alpha-reductase type 2 gene for the diagnosis of $5 \alpha$-reductase deficiency. Journal of Clinical Endocrinology and Metabolism 1996 $813415-3418$

11 ADHR Consortium, Autosomal dominant hypophosphatemic rickets is associated with mutations in FGF23. Nature Genetics 2000 26 345-348.

12 Saggese G, Ranke MB, Saenger P, Rosenfeld RG, Tanaka T, Chaussain JL et al. Diagnosis and treatment of growth hormone deficiency in children and adolescents: towards a consensus. Hormone Research 199850 320-340.
13 Sylvester FA, Shah BR \& Finberg I. Growth hormone stimulation test in familial hypophosphatemia [abstract]. Pediatric Research 199186 A

14 Ogle GD, Rosenberg AR \& Kainer G. Renal effects of growth hormone. II. Electrolyte homeostasis and body composition. Pediatric Nephrology 19926 483-489.

15 Saggese G \& Cesaretti G. Criteria for recognition of the growthinefficient child who may respond to treatment with growth hormone. American Journal of Diseases of Children $1989 \mathbf{1 4 3}$ $1287-1293$

16 Bistritzer T, Chalew SA, Hanukoglu A, Armour KM, Haney PJ \& Kowarski AA. Does growth hormone influence the severity of phosphopenic rickets? European Journal of Pediatrics $19901 \mathbf{1 5 0}$ 26-29.

17 Burgueno M, Malozowski F, Harrison H, Kulin H \& Cassorla F. Growth hormone deficiency in patients with hypophosphatemic rickets [abstract]. Pediatric Research 198823 273A.

18 Ogata T \& Matsuo N. Sex chromosome aberrations and stature: deduction of the principal factors involved in the determination of adult height. Human Genetics 199391 551-562.

19 Lanes R \& Harrison HE. Growth hormone therapy in a poorly growing child with hypophosphatemic rickets. Journal of Endocrinological Investigation 199013 833-837.

20 Jasper $\mathrm{H} \&$ Cassinelli $\mathrm{H}$. Evaluation of the growth hormone somatomedin $\mathrm{C}$ axis in patients with hypophosphatemic rickets. $72 \mathrm{nd}$ Annual Program of the Endocrine Society 1990 300A.

21 Gershberg H, Neumann LL \& Mari S. Studies on vitamin D-resistant rickets; effects of human growth hormone. Metabolism 1964 13 639-649.

22 Saggese G, Baroncelli GI \& Barsanti S. Growth hormone treatment of familial hypophosphatemic rickets. Archives of Pediatrics \& Adolescent Medicie 19985 360S-363S

23 Baroncelli GI, Bertelloni S, Ceccarelli C \& Saggese G. Effect of growth hormone treatment on final height, phosphate metabolism, and bone mineral density in children with X-linked hypophosphatemic rickets. Journal of Pediatrics 2001138 236-243.

24 Kainer G, Nakano M. Massie FS, Foreman JW \& Chan JCM. Hypercalcuria due to combined growth hormone and calcitriol therapy in uremia: effects of growth hormone on mineral homeostasis in $75 \%$ nephrectomized weanling rats. Pediatric Research 199130 $523-533$.

Received 18 March 2003

Accepted 15 July 2003 